\title{
Analysis of Lethality in Echinococcal Disease
}

\author{
Anna S. Khachatryan* \\ Department of Pathological Anatomy and Clinical Morphology, Yerevan State Medical University after M. Heratsi, Yerevan 0025, Republic of Armenia
}

\begin{abstract}
The information on mortality from echinococcosis is important not only for a better understanding of the severity of the disease, but also for evaluating the effectiveness of public health interventions. The aim of this research was to study the causes of mortality from echinococcosis. We have collected and analyzed the materials of 1,470 patients in 10 age - groups in the Republic of Armenia (from 2000 to 2016). To find out the causes of mortality from echinococcosis, we have analyzed the medical histories and protocols of postmortem examinations of 19 deaths from echinococcosis and 17 deaths due to other indirect causes not associated with the parasite. The average annual death rate from echinococcosis is 0.007 per 10,000 population, and the mortality is 1.29 (per 100 patients). The highest mortality occurs in people aged 70-79. Mortality from echinococcosis is also recorded among the unoperated children. The rupture of the parasitic cyst and hepatic insufficiency are major among the direct causes of mortality. Sometimes the hydatid cysts unrecognized during the life were first diagnosed at autopsy. Insufficient qualification of doctors in the field of helminthology, as well as the latent course of the disease or manifestation of minor symptoms in echinococcosis over a long period often led to medical errors. Further decline in mortality can be achieved by early diagnosis, timely hospitalization and treatment before the development of severe complications worsening the prognosis and outcomes of surgical intervention.
\end{abstract}

Key words: Echinococcus granulosus, Echinococcus multilocularis, mortality, echinococcosis, complication

\section{INTRODUCTION}

Hydatid echinococcosis, due to Echinococcus granulosus or E. multilocularis, can occur almost everywhere, but most often in North and East Africa, Asia, South-West Europe, South America, the USA, Canada, Australia, and New Zealand [1-4]. The main causes of death are either complications of hepatic and pulmonary echinococcosis or echinococcosis of the heart. The complications of liver echinococcosis may develop due to the changes occurring not only in the parasitic cyst, but also in the affected organ or in the patient's body. Examples of such complications are: the occurrence of ascites because of the inferior vena cava compression by progressively increasing cyst, the development of fibrinous-purulent peritonitis, the rupture into the bile ducts with obstructive jaundice or biliary-pulmonarybronchial fistulas development. In pulmonary echinococcosis spontaneous pneumothorax, empyema, pericarditis, hepaticbronchial and hepatic-pleural fistulas may occur [5-9].

\footnotetext{
- Received 19 July 2017, revised 3 September 2017, accepted 10 September 2017

*Corresponding author (ann_khachatryan@mail.ru)

(C) 2017, Korean Society for Parasitology and Tropical Medicine

This is an Open Access article distributed under the terms of the Creative Commons

Attribution Non-Commercial License (http://creativecommons.org/licenses/by-nc/4.0) which permits unrestricted non-commercial use, distribution, and reproduction in any

medium, provided the original work is properly cited.
}

Echinococcosis of the heart is a rare localization that occurs in $0.2-2 \%$ cases. Heart lesions are extremely rare without the hepatic involvement. Most frequently the cyst rupture into the pericardium may cause pericardial tamponade, secondary pericardial cysts' development or pericarditis. The rupture of cardiac cysts may cause anaphylactic shock, peripheral and cerebral embolism or pulmonary embolism, dyspnea or right outflow obstruction, pulmonary hypertension and death. Other complications include mitral regurgitation, arrhythmias, coronary syndrome, atrioventricular conduction defects [1014]. The aim of the present study is to determine the causes of death in echinococcosis.

\section{MATERIALS AND METHODS}

We have collected and analyzed the materials of 1,470 patients in 10 age groups from all the hospitals of Armenia. We have used archival materials of surgical departments of these hospitals, reporting documents of sanitary-epidemiological stations, prosectoriums, etc.

For a comprehensive study and generalization of the described processes, methods of epidemiological analysis (retrospective, statistical, medical-geographic, computer, and others) were applied. The results of the investigations were subjected 
to mathematical processing using various statistic methods (paired Student test and Pearson's correlation analysis). To determine the clinical and epidemiological patterns of echinococcosis in Armenia over a period of time (from 2000 to 2016), the average annual morbidity and mortality rates for 10,000 people were calculated.

The data are expressed as mean \pm SD. Pearson's correlation analysis was implemented to determine the statistically significant difference between variables. The statistical analysis was performed by Statistical Package for Social Sciences (SPSS, Chicago, Illinois, USA) version 13.0 for Windows. Statistical significance of differences was set to $P<0.05$. To reveal the causes of mortality in echinococcosis, we have analyzed the medical histories and protocols of postmortem examinations of 19 deaths from echinococcosis.

\section{RESULTS}

According to the results of the epidemiological analysis carried out by us, the occurrence frequency of echinococcosis in women is $0.6 \pm 0.06(n=782)$, whereas in men it is equal to $0.56 \pm 0.06(\mathrm{n}=688)$, but the difference between the indices is not statistically trustworthy $(P>0.05)$. In Armenia, echinococcosis is scattered all over the country. The incidence of echinococcosis among urban population is lower $0.46 \pm 0.04$ $(\mathrm{n}=770)$ than in rural population, the average of which is $0.81 \pm 0.08(\mathrm{n}=700 ; \mathrm{P}<0.001)$.

The age-specific distribution of morbidity from echinococcosis is subject to the following pattern. The highest morbidity was observed in the age groups 50-59 years, $0.82 \pm 0.18$ $(\mathrm{n}=152), 60-69$ years, $0.73 \pm 0.16(\mathrm{n}=145)$, and $11-16$ years, $0.64 \pm 0.13(n=164)$. The morbidity decreases after 70 years of age, $0.48 \pm 0.16(n=66 ; P<0.001)$. The incidence of echinococcosis in patients from 1 year to 18 years is $0.48 \pm 0.06$ $(\mathrm{n}=378)$. The highest incidence rate was estimated among children aged 11-16 years $0.64 \pm 0.13(n=164)$. It may be thought that a rarer detection of echinococcosis in children under 7 years of age $(0.23 \pm 0.1, \mathrm{n}=35)$ depends on the fact that the disease is still in the latent period and is manifested in especially severe cases of complications. The youngest patient observed among our patients was a child of 3 years old.

Echinococcosis can affect any organ, although it is established that most often the parasitic cyst was localized in the liver, amounting to $59.5 \% \pm 1.23(\mathrm{n}=947)$, in the lungs $33.1 \% \pm 1.18(n=526)$, the remaining rare localizations of echinococcus in general are $7.4 \% \pm 0.66(\mathrm{n}=118)$. In women the liver is affected more often by echinococcosis $-0.42 \pm 0.05$ $(\mathrm{n}=551)$ than in men $-0.32 \pm 0.04(\mathrm{n}=396)$. The difference between the indices is statistically trustworthy $(P=0.05)$. The number is more than 1,470 , because of multiple echinococcosis cases (i.e. in some patients multiple echinococcal cysts were revealed in the liver, lungs, spleen).

Among the 19 deaths from echinococcosis there were 10 women (52.6\%) and 9 men (47.4\%). Concomitant diseases were not revealed in any these of cases. The highest mortality was observed in people aged 70-79. Mortality was also recorded among the unoperated children (with echinococcosis of the heart, 1 child; with lung echinococcosis, 1 child). The average annual mortality rate is $P=0.007$, and the lethality rate was $P=1.29$ per 100 patients. These indicators not only characterize the severity of the clinical course of the disease, but also allow to judge indirectly the effectiveness of public health interventions.

By the localization of the hydatid cyst in organs of the dead, the first place is the liver, the second is the lungs, and then rare localizations. Data about the localization of hydatid cysts in the organs of dead patients are shown in Table 1. Simultaneous lesion of 2 or 3 organs (lungs and liver; lungs, liver, and heart; liver and heart; liver and spleen) was noted in 3 deaths.

The death from the parasitic cyst spontaneous rupture in our material was detected in 5 patients $(26.3 \%)$. This severe complication caused by the parasitic cyst integrity break and the spill of its contents into the cavity of the host organism (pleural, peritoneal, and pericardium), gave rise to new even more severe complications. In this group, anaphylactic shock occurred in 2 patients and the rupture of the suppurated cyst causing severe septic complications (peritonitis, pleurisy, and pericarditis) in 3 patients. For instance, a 15-year-old girl suddenly lost consciousness during the meal and died in a reanimobile on the way to hospital. During the autopsy a giant ruptured hydatid cyst of the right lung was discovered (Fig. 1).

Five deaths (26.3\%) were associated with the suppuration

Table 1. Organ localization of echinococcal cysts in the deads from echinococcosis

\begin{tabular}{lrr}
\hline Name of the organ & No. of observations & \% ratio \\
\hline Liver & 14 & 63.6 \\
Lungs & 5 & 22.7 \\
Heart & 2 & 9.1 \\
Pericardium & 1 & 4.6 \\
Total & 22 & 100.0 \\
\hline
\end{tabular}




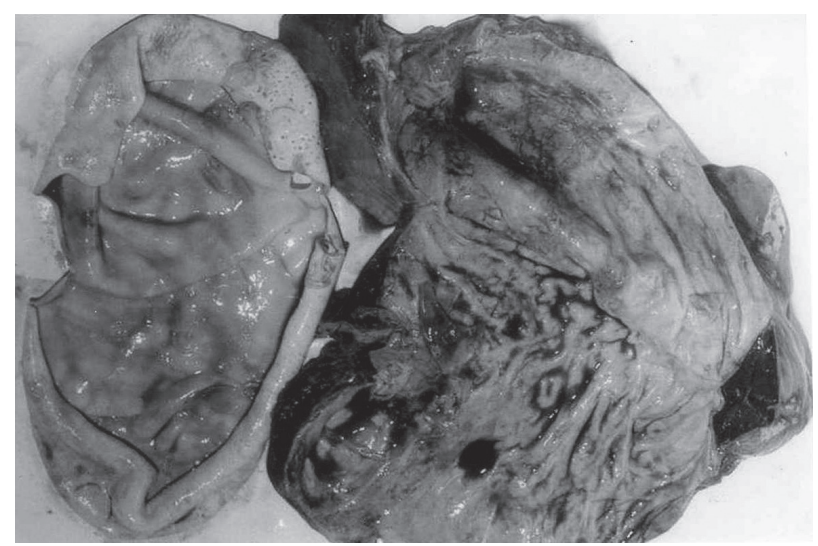

Fig. 1. Ruptured giant echinococcal cyst of the right lung $(25 \times 15$ $\times 15 \mathrm{~cm}$ in size) with the subtotal replacement of pulmonary parenchyma.

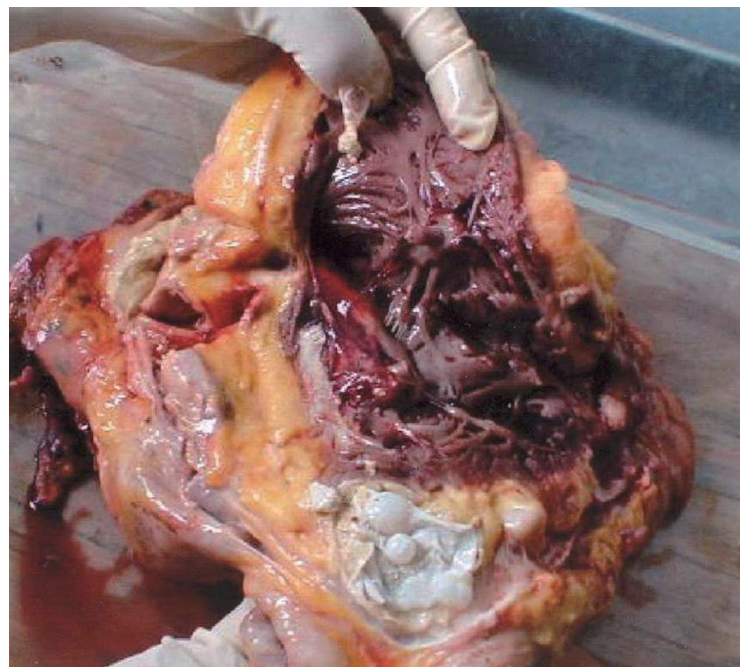

Fig. 2. Multiple suppurated and ruptured echinococcal cysts of the pericardium due to extensive intrapericardial dissemination with the pericardial cavity obliteration and pericarditis development.

of hydatid cysts which caused purulent intoxication followed by septic state. One of the causes of death in echinococcosis is hepatic failure, which was observed in 9 patients (47.4\%). The patients of this group, being in incurable condition, did not even undergo surgery, they received only conservative treatment. The number of rupture, suppuration and hepatic failure cases is more than 19, because in some cases all the complications were met only in 1 person (i.e. rupture and suppuration, rupture and hepatic failure or rupture of the heart echinococcal cyst).

Other causes of sudden deaths were heart lesions (echinococcosis of the heart, pericardium). The following observations are brought as an illustration. A 51-year-old man had

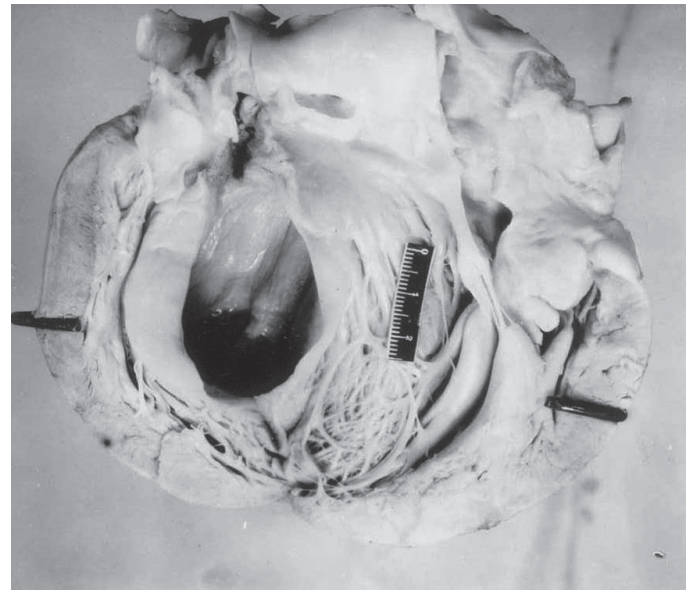

Fig. 3. Ruptured echinococcal cyst $(6 \times 4 \times 3.5 \mathrm{~cm}$ in size $)$ in the heart interventricular septum.

been complaining of chest pains for many years and was unsuccessfully treated by cardiologists for ischemic heart disease. Within 2 days the patient's condition suddenly deteriorated and death occurred. The autopsy discovered multiple pericardial hydatid cysts, the rupture of which led to the development of pericardial cavity obliteration, pericarditis, and pleural empyema of the left lung (Fig. 2). The case of heart echinococcosis in a 17-year-old sportsman, who had never complained of his poor health. The sudden death occurred during the routine running. A ruptured hydatid cyst in the interventricular septum of the heart (Fig. 3) was detected during the postmortem examination.

In all the aforesaid cases, the diagnosis of echinococcosis was confirmed histologically upon detection of chitinous and fibrous membranes of the wall. The autopsies have also discovered interesting and rare combinations of liver echinococcosis with lung cancer and pleomorphic sarcoma in the retroperitoneum.

\section{DISCUSSION}

Echinococcosis of 19 deaths was identified during autopsy not as a concomitant disease, but as a direct cause. Seventeen deaths due to other indirect causes (combinations of malignant tumors with echinococcosis) not associated with the parasite were not included either in analysis or accounting mortality and lethality rates. These combinations are most likely due to not only the prolonged chronic inflammatory process and toxic effect of hydatid cysts on the host organism but also due to immunosuppressants secreted by echinococcal cysts 
and contributing to the development of malignant tumors.

The results concerning the lethality rate $(P=1.29$ per 100 patients) coincides with the data obtained by Belhassen-García et al. [15] (1.94\%), the main causes of which were the complications related to the rupture of echinococcal cysts with supurative cholangitis. We have detected a higher incidence among the rural population, but the results of the investigations conducted by Chaâbane-Banaoues et al. [16] have shown that the pathogen dissemination is influenced by human activities and behavior towards the infected viscera. Our results don't show male predominance among the 19 deaths in contrast to the studies of some other authors $[17,18]$, who have described the male predominance of mean age 31-33.2 years in their works.

The rupture of the parasitic cyst and hepatic insufficiency are the major direct causes of mortality. The rupture of echinococcal cysts led to anaphylactic shock due to the leakage of cystic fluid into the bloodstream and intravascular spread of the cyst contents. The rupture was spontaneous in all cases, it was not preceded by trauma. But as shown in M. Hosseini's et al. work the sudden death was due to an intact hydatid cyst in a 13-year-old girl, caused by the anaphylactic shock after a blunt abdominal trauma [19]. The hepatic failure developed both due to the parasitic cirrhosis of the liver and the prolonged compression of bile ducts by the parasite.

Heart lesions by echinococcosis were presented as rare cases in the clinical material among thousands of observations, whereas they were more common in the autopsy material. Although many authors in their works [10-14] have reported about the expressed clinical symptoms of heart echinococcosis (e.g. dyspnea, chest pain) even during several months in our material it lasted a long period without any symptoms. The abovementioned examples are vivid illustrations of strong compensatory features of the heart, which concealed this foreign formation over years. Only 1 patient was unsuccessfully treated by cardiologists for ischemic heart disease. The coronary syndrome in this case was secondary due to the coronary artery compression by the hydatid cyst. The asymptomatic clinical presentation of cardiac echinococcal cysts most likely depended on the small size of cysts and absence of complications resulted from cyst rupture. Only after rupture it led to anaphylactic shock due to the leakage of cystic fluid into the ventricular cavity, complete atrioventricular block because of the localization in the interventricular septum, effusion with the pericardial cavity obliteration and pericarditis development.
In 15 of 19 deaths, echinococcosis and its complications were not diagnosed during the lifetime. In the majority of these cases the causes of the severe complications were only clarified during autopsy. Fatal outcomes were not observed in cases of early surgery in the uncomplicated stages of the disease. Although hydatid cysts can occur in most atypical localizations, in case of certain suspicion it is possible to establish the precise localization of the hydatid cyst by simple radiological investigation methods even in the asymptomatic stage of the disease. The atypical course of echinococcosis should be taken into consideration too, especially in the endemic regions, i.e. the pain radiation to the epigastrium and mimicking an acute abdomen [20], false impression of ventricular aneurysm [21], coronary artery disease.

Thus, the analysis of our material shows that the main cause of death from echinococcosis is the patients' late hospital admission and a very high percentage of diagnostic errors in complicated echinococcosis. The main reason of neglected echinococcosis is unawareness of clinical symptoms of the disease, which requires active propaganda among the population. This is of special importance in the regions where echinococcosis is most prevalent.

\section{ACKNOWLEDGMENTS}

We thank YSMU for administrative support. We thank Dr. Sergey A. Urumyan, Associate Professor, Chief Executive Officer of MC "Shengavit" for technical support. This research work is supported by Yerevan State Medical University, Republic of Armenia.

\section{CONFLICT OF INTEREST}

We declare that we have no competing interests. Informed consent was obtained from all individual participants included in the study.

\section{REFERENCES}

1. Amado-Diago CA, Gutiérrez-Cuadra M, Armiñanzas C, Arnaíz de Las Revillas F, Gómez-Fleitas M, Fariñas MC. Echinococcosis: a 15-year epidemiological, clinical and outcome overview. Rev Clin Esp 2015; 215: 380-384.

2. Craig PS, Hegglin D, Lightowlers MW, Torgerson PR, Wang Q. Echinococcosis: control and prevention. Adv Parasitol 2017; 96: 55-158. 
3. Brundu D, Piseddu T, Stegel G, Masu G, Ledda S, Masala G. Retrospective study of human cystic echinococcosis in Italy based on the analysis of hospital discharge records between 2001 and 2012. Acta Trop 2014; 140: 91-96.

4. Melia K, Kokaia N, Manjgaladze M, Kelbakiani-Kvinikhidze T, Sulaberidze G. Clinical and epidemiological peculiarities of cystic echinococcosis in children. Georgian Med News 2017; 265 : 79-83 (in Russian).

5. Pakala T, Molina M, Wu GY. Hepatic echinococcal cysts: a review. J Clin Transl Hepatol 2016; 4: 39-46.

6. Kern P, Menezes da Silva A, Akhan O, Müllhaupt B, Vizcaychipi KA, Budke C, Vuitton DA. The echinococcoses: diagnosis, clinical management and burden of disease. Adv Parasitol 2017; 96: 259-369.

7. Sarkar S, Roy H, Saha P, Sengupta M, Sarder K, Sengupta M. Cystic echinococcosis: a neglected disease at usual and unusual locations. Trop Parasitol 2017; 7: 51-55.

8. Taxy JB, Gibson WE, Kaufman MW. Echinococcosis: unexpected occurrence and the diagnostic contribution of routine histopathology. Am J Surg Pathol 2017; 41: 94-100.

9. Lianos GD Lazaros A, Vlachos K, Georgiou GK, Harissis HV, Mangano A, Rausei S, Boni L, Frattini F, Biondi A, Dionigi G, Katsios C. Unusual locations of hydatid disease: a 33 year's experience analysis on 233 patients. Updates Surg 2015; 67: 279282.

10. Shojaeifard M, Hosseini S, Hosseini S, Pouraliakbar H, Dehghani H, Noohi F. Cardiac hydatid cyst without liver involvement: a case report. Iran J Parasitol 2016; 11: 274-278.

11. Padmanabhan TNC, Kumar KVK, Sadiq Azam M, Kumar Bilolikar A. Primary Echinococcus infection of the heart: a rare type of cystic echinococcosis. Eur Heart J 2017; 38: 2255.

12. Qadri SK, Hamdani NH, Bhat AR, Lone MI. Unusual presenta- tion of an intraventricular hydatid cyst as a bleeding cystic tumor: a case report and brief review. Asian J Neurosurg 2017; 12: 324-327.

13. Sabzi F, Vaziri S, Faraji R. Right ventricular hydatid cyst ruptured to pericardium. Ann Card Anaesth 2015; 18: 445-448.

14. Sabzi F, Faraji R. A giant hydatid cyst in the interventricular septum with papillary muscle involvement. Korean J Parasitol 2013; 51: 349-352.

15. Belhassen-García M, Romero-Alegria A, Velasco-Tirado V, Alonso-Sardón M4, Lopez-Bernus A, Alvela-Suarez L, del Villar LP, Carpio-Perez A, Galindo-Perez I, Cordero-Sanchez M, PardoLledias J. Study of hydatidosis-attributed mortality in endemic area. PLoS One 2014; 9: e91342.

16. Chaâbane-Banaoues R, Oudni-M'rad M, M'rad S, Mezhoud H, Babba H. Environmental contamination by Echinococcus granulosus sensu lato eggs in relation to slaughterhouses in urban and rural areas in Tunisia. Korean J Parasitol 2016; 54: 113-118.

17. Jedidi M, Mlayeh S, Masmoudi T, Souguir MK, Zemni M. Sudden death due to hydatid cyst: thirty-four medicolegal autopsy cases. Am J Forensic Med Pathol 2014; 35: 29-33.

18. Ben Khelil M, Allouche M, Banasr A, Gloulou F, Benzarti A, Zhioua M, Haouet S, Hamdoun M. Sudden death due to hydatid disease: a six-year study in the northern part of Tunisia. J Forensic Sci 2013; 58: 1163-1170.

19. Hosseini M, Hedjazi A, Bahrami R. Sudden death due to anaphylactic shock in a patient with an intact hepatic hydatid cyst. Am J Forensic Med Pathol 2014; 35: 256-257.

20. Dighiero J, Canabal EJ, Aguirre CV, Hazan J, Horjales JO. Echinococcus disease of the heart. Circulation 1958; 17: 127-132.

21. Uysalel A, Aral A, Atalay S, Akalin H. Cardiac echinococcosis with multivisceral involvement. Pediatr Cardiol 1996; 17: 268270 . 
